2. To: (Receiving Organization)

DISTRIBUTION

5. Proj./Prog./Dept./Div.:

INTERIM STABILIZATION

8. Originator Remarks:

ATTACHED IS A GUIDANCE FOR THE PROGRAMMING OF THE FLOW METER AND DIGITAL DISPLAYS ON THE PIC SKIDS.

11. Receiver Remarks:

NONE
3. From: (Originating Organization)

INTERIM STABILIZATION

6. Design Authority/Design Agent/Cog. Engr.:

W. F. ZUROFE
11A. Design Baseline Document? $\bigotimes$ Yes $\square$ No
4. Related EDT No.:

N/A

7. Purchase Order No.:

$\mathrm{N} / \mathrm{A}$

9. Equip./Component No.:

$\mathrm{N} / \mathrm{A}$

10. System/Bidg./Facility:

$241 \mathrm{G}$

12. Major Assm. Dwg. No.:

$\mathrm{N} / \mathrm{A}$

13. Permit/Permit Application No.:

$\mathrm{N} / \mathrm{A}$

14. Required Response Date:

$06 / 30 / 00$

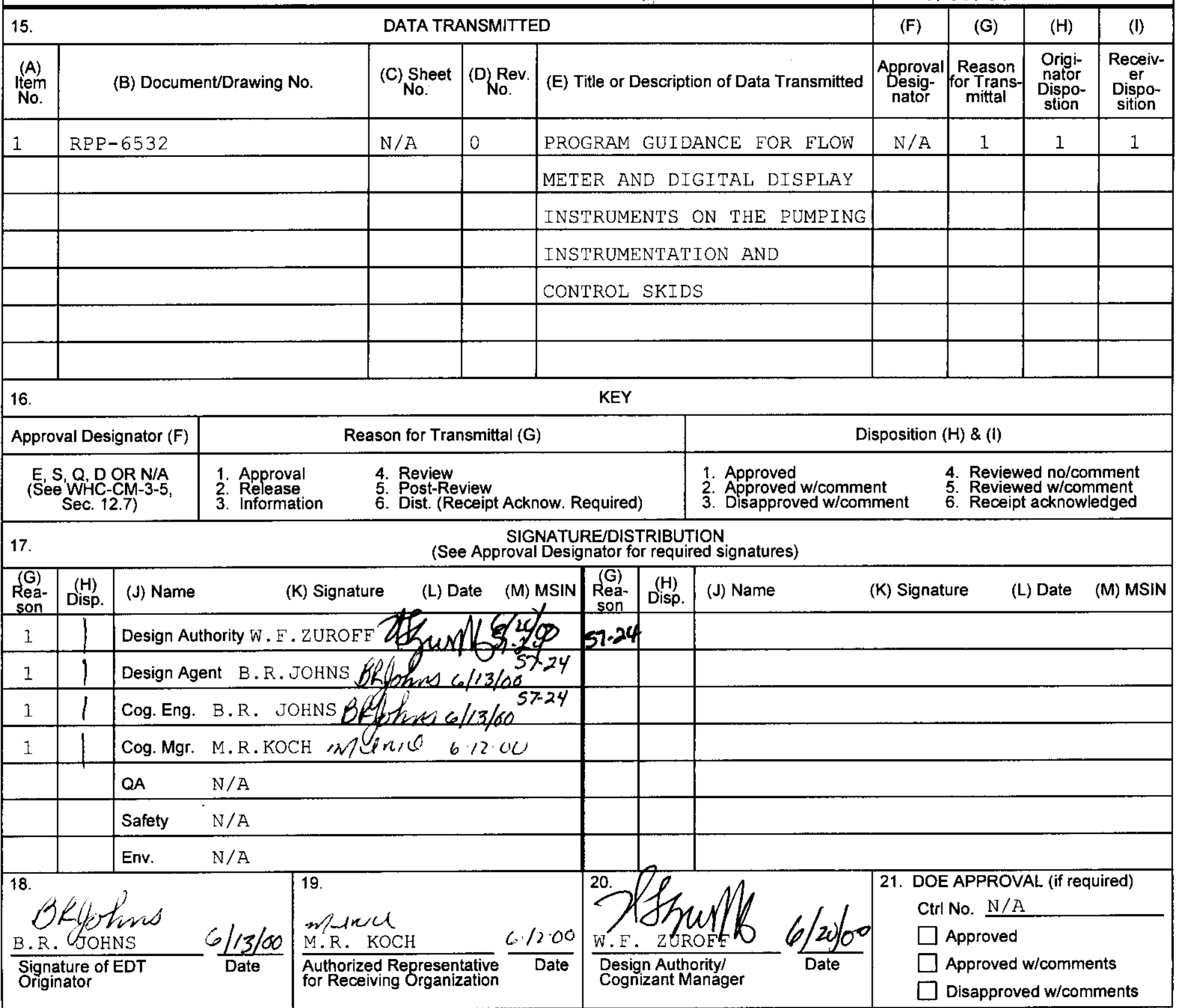




\title{
PROGRAM GUIDANCE FOR FLOW METER AND DIGITAL DISPLAY INSTRUMENTS ON THE PUMPING INSTRUMENTATION AND CONTROL SKIDS
}

\author{
M. R. KOCH \\ CH2MHILL HANFORD GROUP, INC \\ Richland, WA 99352 \\ U.S. Department of Energy Contract DE-AC06-99RL14047 \\ EDT/ECN: $629884 \quad$ UC: \\ Cost Center: 74D00 Charge Code: 103361 \\ B\&R Code: EW3120071 Total Pages: 8 \\ Key Words: INSTRUMENTATION, PICS, SALT WELL, SKID, INTERIM STABILIZATION \\ PROGRAMMING

\begin{abstract}
:
This guidance is for the programming of the Yokogawa flow meter and Yokogawa digital displays on the PIC skids.
\end{abstract}

TRADEMARK DISCLAIMER. Reference herein to any specific commercial product, process, or service by trade name, trademark, manufacturer, or otherwise, does not necessarily constitute or imply its endorsement, recommendation, or favoring by the United States Government or any agency thereof or its contractors or subcontractors.

Printed in the United States of America. To obtain copies of this document, contact: Document Control Services, P.O. Box 950, Mailstop H6-08, Richland WA 99352, Phone (509) 372-2420; Fax (509) 376-4989.
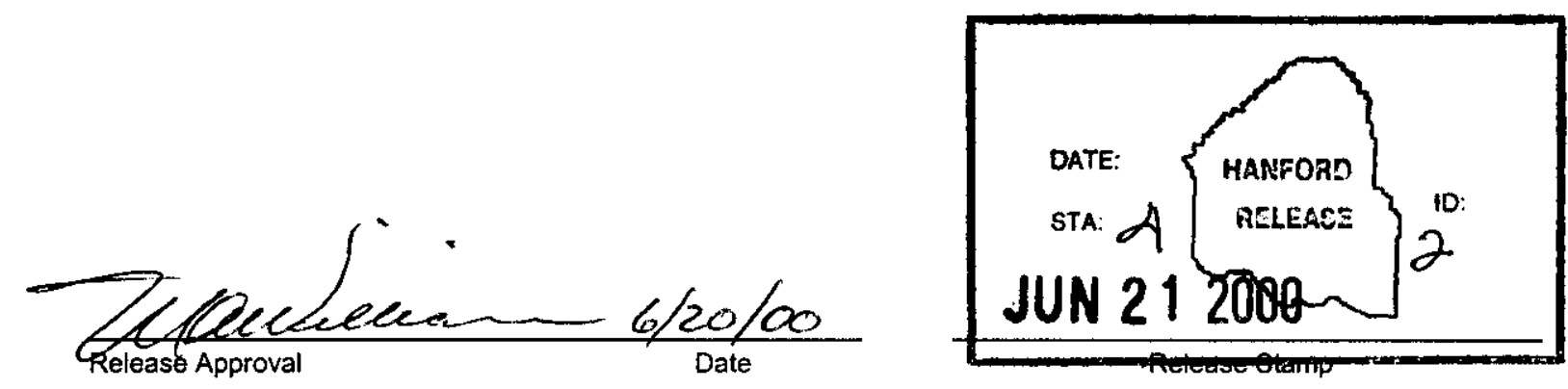

\section{Approved For Public Release}




\section{PROGRAM GUIDANCE FOR FLOW METER AND DIGITAL DISPLAYS ON PUMPING INSTRUMENTATION AND CONTROL SKIDS}

\section{INTRODUCTION}

The Pumping Instrumentation and Control Skids (PICS) contain a flow meter to display the flow in the discharge line and two digital displays to show the pressure in the suction and discharge lines of the jet pump. These three instruments require programming during initial setup. Programming consists of inputting parameters to fit the field application of the instruments.

\section{SCOPE}

This guidance document provides the initial programming parameters for the Yokogawa AE14 flow meter and the Yokogawa UM330 digital display units. The guidance applies only to initial programming and may be changed in the field during setup of the PICS at specific tanks.

\section{FLOW METER PROGRAM}

The Yokogawa flow meter is located in the Instrument Enclosure on the PIC skid. Appendix A provides a list of the programming parameters for the flow meter. Programming of the flow meter can be performed using a "Brain terminal" or by using the three white buttons on the front of the unit. The top portion of the chart in Appendix A identifies the parameters that must be entered to setup the flow meter for calibration and field use. The bottom portion of the chart contain parameters that normally remain at factory settings.

The shaded portion of the program chart contains the displays and settings when using the three white buttons on the face of the flow meter. Note there are some settings that are not available when using the white buttons, but are available when using the brain terminal. Engineering will provide the high and low meter factors. Reference the vendor's manual for AE14 Flow meters for instructions as to the operation of the white buttons or the brain terminal.

Appendix A contains a sample printout of the flow meter parameters. This printout is only available when using the brain terminal. The "L01" parameter allows the programmer to "disable" the parameters from being changed once setup.

\section{DIGITAL DISPLAY PROGRAMMING}




\section{RPP-6532 \\ REVISION 0}

Appendix B contains the programming chart for the digital displays that monitor the suction and discharge pressure. These two displays require programming prior to calibration. The chart in Appendix B lists the parameters and values to be entered. The parameters on the bottom portion of the chart should not require changing and should be left with the factory settings. Reference the vendor's manual for the UM330 for details as how to enter the program parameter settings. 
RPP-6532

REVISION 0

\section{APPENDIX A: YOKOGAWA FLOW METER PROGRAM}

\begin{tabular}{|c|c|c|c|c|}
\hline \multicolumn{5}{|c|}{ YOKOGAWA FLOW METER PROGRAM VALUES TO BE SET } \\
\hline $\begin{array}{l}\mathrm{LCD} \\
\text { DISPLAY }\end{array}$ & SETTINGS & $\begin{array}{c}\text { BRAIN } \\
\text { TERMINAL }\end{array}$ & SETTINGS & DESCRIPTION \\
\hline & & $\mathrm{B} 01$ & $6001 @$ & Tag no. ((d) $=$ skid letter $)$ \\
\hline 02 & 10 & $\mathrm{~B} 02$ & 1.0 & Time constant, $1 \mathrm{sec}$. \\
\hline 03 & $14.000 \#$ & $\mathrm{BO} 3$ & 14.0000 & Span \\
\hline 04 & 06 & B04 & GAL & Gallons \\
\hline 05 & 02 & B05 & $\mathrm{M}$ & Minutes \\
\hline 06 & 01 & B 06 & $\mathrm{INCH}$ & Inches \\
\hline 07 & 0.5 & $\mathrm{~B} 07$ & 0.5 & Flow tube nominal size \\
\hline 08 & $*$ & B0) 8 & $*$ & Low meter factor \\
\hline 09 & 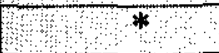 & $\mathrm{B} 09$ & $*$ & High meter factor \\
\hline 10 & 1 & $\mathrm{~B} 10$ & $\begin{array}{l}\text { ALARM } \\
\text { OUT }\end{array}$ & Output function \\
\hline 12 & 60.0 & $\mathrm{~B} 12$ & 60.0 & Power frequency \\
\hline d] & 06 & Dol & $\begin{array}{c}\mathrm{RATE} / \mathrm{FOR} \\
\text { TTL }\end{array}$ & Display sclect \\
\hline $\mathrm{E} 1$ & 03 & $\mathrm{E} 01$ & $\mathrm{UNIT} / \mathrm{P}$ & Totalizer units \\
\hline E2 & 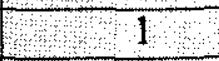 & $\mathrm{E}(0)$ & 1 & Multiplier of E2(1 gpm) \\
\hline $\mathrm{E} 3$ & 1 & $\mathrm{E} 03$ & 1 & Minimum per cent reading \\
\hline
\end{tabular}

\# Engineering to provide actual span value, normally 8 or 14 gpm.

* Values taken from llow head to be used with llow meter. 
RPP-6532

REVISION 0

\begin{tabular}{|c|c|c|c|c|}
\hline \multicolumn{5}{|c|}{ YOKOGAWA FLOW METER PROGRAM FACTORY SETTINGS } \\
\hline LCD & SETTINGS & BRAIN & SETTINGS & DESCRIPTION \\
\hline DISPLAY & & TERMINAL & & \\
\hline 11 & 00 & $\mathrm{~B} 11$ & $\begin{array}{l}2.4 \mathrm{~mA} \text { or } \\
\text { I.ESS }\end{array}$ & 4-20 ALM OUT \\
\hline 13 & $\begin{array}{l}\text { (computer } \\
\text { generated) }\end{array}$ & $\mathrm{B} 13$ & $\begin{array}{l}\text { (computer } \\
\text { generated) }\end{array}$ & $\begin{array}{c}\text { (NOT USED WHEN BOG SET } \\
\text { TO INCHES) }\end{array}$ \\
\hline 14 & 00 & $\mathrm{~B} 14$ & FORWARD & FLOW DIRECTION \\
\hline 30 & 10.000 & $\mathrm{~B} 30$ & $10.000 \mathrm{G} \wedge \mathrm{L} / \mathrm{M}$ & REV.SPAN \\
\hline 31 & 2 & $\mathrm{~B} 31$ & 2 & DIREC HYS \% \\
\hline 33 & 10.000 & B33 & $10.000 \mathrm{GAL} / \mathrm{M}$ & FOR. SPAN2 \\
\hline 34 & 10 & $\mathrm{~B} 34$ & 10 & $\mathrm{HY}^{\prime} \mathrm{S}$ \\
\hline 36 & 10 & B36 & -10 & LOW ALARM \% \\
\hline 37 & 5 & B37 & 5 & L. ALARM HYS \% \\
\hline $\mathrm{Cl}$ & 01 & $\mathrm{COI}$ & ENABLE & ZERO TUNING \\
\hline $\mathrm{C} 2$ & 0.02 & $\mathrm{CO} 2$ & 0.02 & MAGFLOW ZERO \\
\hline $\mathrm{d} 2$ & 00 & $\mathrm{D} 02$ & $\begin{array}{c}\text { NOT } \\
\text { PROVIDED }\end{array}$ & $\begin{array}{l}\text { DISPLAY PANEL } \\
\text { SELECTION }\end{array}$ \\
\hline d3 & 100 & Do3 & 100 & USER SPAN \\
\hline & 4 & D10 & (SPACE) & USER UNIT \\
\hline $\mathrm{E4}$ & 01 & $\mathrm{E}(04$ & ENABLE & TOTAL SET \\
\hline $\mathrm{ES}$ & 0 & $\mathrm{E} 05$ & 0 & TL SET VALUE \\
\hline E6 & 0 & E06 & () & TOTAL SWITCH \\
\hline & 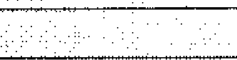 & E 10 & (SPACE) & TI USER UNIT \\
\hline & 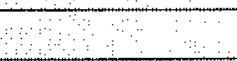 & E11 & () & REV. TOTAL \\
\hline & & $\mathrm{E} 12$ & -1 & DIF. TOTAL \\
\hline G1 & 0 & Gol & 0 & $4-20 \mathrm{LOOW}$ CUT \\
\hline G2 & 20 & $\mathrm{G} 02$ & -20 & 4-20 LOW LMT \\
\hline G3 & 120 & $\mathrm{G} 03$ & 120 & 4-20 H LMT \\
\hline $\mathrm{HI}$ & $00^{* *}$ & $\mathrm{HOI}$ & NORMAL ${ }^{* *}$ & TEST MODE \\
\hline $\mathrm{H2}$ & ** & $\mathrm{HO2}$ & $* *$ & OUTPUT VALUE \\
\hline $\mathrm{H3}$ & 00 & $\mathrm{HO}$ & NORMAL & STATUS OUT \\
\hline L1 & $01^{* * *}$ & Lol & ENABLE*** & TUNING \\
\hline 12 & 55 & $\mathrm{~L}() 2$ & 55 & $\mathrm{KEY}$ \\
\hline $\mathrm{ni}$ & 01 & Nol & DAMP & TOTAL/PAUSE \\
\hline $\mathrm{n} 2$ & 00 & $\mathrm{~N}() 2$ & $\mathrm{ON}$ & OUTPUT MODE \\
\hline$n 3$ & 5 & $\mathrm{No3}$ & 5 & RATE LIMIT \\
\hline $\mathrm{n} 4$ & 0 & $\mathrm{No4}$ & 0 & DEAD TIME \\
\hline $\mathrm{n} 5$ & 00 & N05 & YES & POWER SYNCH \\
\hline $\mathrm{n} 6$ & 01 & No6 & $\mathrm{NO}$ & PULSING FLOW \\
\hline $\mathrm{n} 7$ & 00 & N07 & ALARM & EMPTY PIPE \\
\hline
\end{tabular}

** For test mode, $\mathrm{H} 1$ is changed to "TEST" $(00)$ and $\mathrm{H} 2$ is set to percent of output desired.

*** (hange to "DISABLE:" $(00)$ to prevent unauthorized dita changes. 
RPP-6532

REVISION 0

EXAMPLE YOKOGAWA FLOW METER PROGRAM PRINTOUT

TITLE: 6001P

DATE 06-08-00 TIME 08:00

A10: FLOW RATE (\%)

$$
-10.0 \%
$$

A20: FLOW RATE $-0.800 \quad \mathrm{gal} / \mathrm{m}$

A30: TOTAL

0.

A60: SELF CHECK ERROR

BO1: TAG NO

$6001 P$

B02: DAMPING

$1.0 \mathrm{Sec}$

B03: FLOW SPAN

$14.000 \mathrm{gal} / \mathrm{m}$

Gal

B05: TIME UNIT

B06: SIZE UNIT

inch

B07: NOMINAL SIZE

B08: LOW MF 0.50 inch

1.3052

B09: HIGH MF

$$
1.2140
$$

B10: OUTPUT FUNC

$$
\text { ALARM OUT }
$$

B11: 4-20 ALM OUT

2.4mA OR LESS

B12: POWER FREQ $60.01 \mathrm{~Hz}$

B13: VELOCITY CHK $3.984 \mathrm{~m} / \mathrm{s}$

B14: FLOW DIR FORWARD

B30: REV. SPAN $10.000 O \mathrm{gal} / \mathrm{m}$

B31: BI DIREC HYS 2. $\%$

B33: FOR. SPAN2 $10.000 \mathrm{gal} / \mathrm{m}$
B34: AUTO RNG HYS

10. $\%$

B36: LOW ALARM

$10 . \%$

B37: L. ALARM HYS

5. $\%$

B60: SELF CHECK

ERROR

C01: ZERO TUNING

ENABLE

C02: MAGFLOW ZERO 0.02

C60: SELF CHECK ERROR

D01: DISP SELECT

RATE/FOR. TTL

D02: FL USER SEL NOT PROVIDED

D03: FL USER SPAN

$$
100.0
$$

D10: FL USER UNIT

D60: SELF CHECK

ERROR

E01: TOTAL UNIT UNIT/P

E02: TOTAL SCALE 1.0 UNIT/P

E03: TOTAL LOWCUT

E04: TOTAL SET ENABLE

E05: 'TL SET VALUE

0.

E06: TOTAL SWITCH

0 .

E10: TL USER UNIT

E11: REV. TOTAL

0.

E12: DIF. TOTAL

-1 .
E60: SELF CHECK ERROR

G01: 4-20 LOW CUT

$0 . \%$

G02: 4-20 LOW LMT

- 2O\%

G03: 4-20 H LMT

120. $\%$

G60: SELF CHECK ERROR

H01: TEST MODE NORMAL

H02: OUTPUT VALUE

$0 . \%$

H03: STATUS OUT NORMAL

H60: SELF CHECK

ERROR

L01: TUNING

ENABLE

L02: KEY

55.

L60: SELF CHECK ERROR

N01: TOTAL/PULSE DAMP

N02: OUTPUT MODE ON

N03: RATE LIMIT

$5 . \%$

N04: DEAD TIME

N05: POWER SYNCH YES

N06: PULSING FLOW

No

N07: EMPTY PIPE ALARM

N60: SELF CHECK ERROR 
RPP-6532

REVISION 0

\section{APPENDIX B: DIGITAL DISPLAY PROGRAM}




\section{RPP-6532 \\ REVISION 0}

\begin{tabular}{|c|c|c|}
\hline \multicolumn{3}{|c|}{ YOKOGAWA DIGITAL DISPLAY PROGRAM SETTINGS } \\
\hline SYMBOL & DESCRIPTION & PROGRAM ENTRY \\
\hline \multicolumn{3}{|c|}{ Set the following parameters first. } \\
\hline$\overline{\mathrm{IN}}$ & Input type & 41 \\
\hline$\overline{\text { UNIT }}$ & Input unit & N/A \\
\hline$\overline{\mathrm{RH}}$ & Maximum measured range input & 5 \\
\hline RL & Minimum measured range input & 1 \\
\hline SDP & Decimal point position & 1 \\
\hline SH & Input scale upper limit & $\begin{array}{c}300 \text { (discharge); } \\
100 \text { (suction) }\end{array}$ \\
\hline$\overline{\mathrm{SL}}$ & Input scale lower limit & 0 \\
\hline RJC & Input RJC & ON \\
\hline$\overline{\mathrm{BSL}}$ & Input burnout operation selection & 1 \\
\hline RET & Retransmission output & 1 \\
\hline RTH & Maximum retransmission & N/A \\
\hline RTL & Minimum retransmission & N/A \\
\hline$\overline{\mathrm{DIS}}$ & DI function selection & 1 \\
\hline C.S1 & Display 1 & OFF \\
\hline C.S2 & Display 2 & OFF \\
\hline C.S3 & Display 3 & $\mathrm{OFF}$ \\
\hline C.S4 & Display 4 & OFF \\
\hline LOCK & Keylock & OFF \\
\hline PWD & Password & 1 \\
\hline \multicolumn{3}{|c|}{ The parameters below remain with factory default settings. } \\
\hline A1 & Alarm 1 & 1 \\
\hline $\mathrm{A} 2$ & Alarm 2 & 2 \\
\hline $\mathrm{A} 3$ & Alarm 3 & 1 \\
\hline A4 & Alarm 4 & 2 \\
\hline PEAK & Maximum value of $P V$ & N/A \\
\hline BOTM & Minimum value of PV & N/A \\
\hline FL & Input filter & Off \\
\hline BS & Input bias & 0 \\
\hline HY1 & Alarm 1 hysteresis & 0.5 \\
\hline HY2 & Alarm 2 hysteresis & 0.5 \\
\hline HY3 & Alarm 3 hysteresis & 0.5 \\
\hline HY4 & Alarm 4 hysteresis & 0.5 \\
\hline P.SL & Protocol selection & 0 \\
\hline BPS & Comm. Speed & 4 \\
\hline STP & Stop bit & 1 \\
\hline DLN & Data length & 8 \\
\hline $\mathrm{ADR}$ & \begin{tabular}{|l|l} 
Address \\
\end{tabular} & 1 \\
\hline RP.T & Minimum response time & 0 \\
\hline
\end{tabular}

